\title{
Ultra-lightweight Chemical Vapor Deposition grown multilayered graphene coatings on paper separator as interlayer in lithium-sulfur batteries
}

\author{
Elif Ceylan Cengiz a, b, Omer Salihoglu ${ }^{\text {c, d }}$, Osman Ozturk b, e, Coskun Kocabas c, f, \\ Rezan Demir-Cakan ${ }^{\text {b, g, * }}$ \\ a Department of Material Science and Engineering, Gebze Technical University, 41400, Gebze, Kocaeli, Turkey \\ ${ }^{\mathrm{b}}$ Institute of Nanotechnology, Gebze Technical University, 41400, Gebze, Kocaeli, Turkey \\ c Department of Physics, Bilkent University, 06800, Ankara, Turkey \\ d Energy Institute, TUBITAK Marmara Research Center, 41470, Gebze, Kocaeli, Turkey \\ e Department of Physics, Gebze Technical University, 41400, Gebze, Kocaeli, Turkey \\ ${ }^{\mathrm{f}}$ School of Materials, National Graphene Institute, University of Manchester, M13 9PL, Manchester, United Kingdom \\ ${ }^{g}$ Department of Chemical Engineering, Gebze Technical University, 41400, Gebze, Kocaeli, Turkey
}

\section{A R T I C L E I N F O}

\section{Article history:}

Received 10 September 2018

Received in revised form

4 November 2018

Accepted 6 November 2018

Available online 9 November 2018

\section{Keywords:}

Lithium-sulfur batteries

Anode protection

Lithium metal

Interlayer

Graphene

\begin{abstract}
A B S T R A C T
Lithium-Sulfur (Li-S) batteries are known for their high energy density and cost-effectiveness. However, the Li-S chemistry is a challenging topic due to the shuttle effect and the use of highly reactive lithium metal anode. To solve these issues, ultra-lightweight multilayered graphene coated paper separator is proposed as an interlayer. The interlayer is firstly coated on nickel foil by Chemical Vapor Deposition (CVD) method followed by transferring to the paper by fishing process. By employing this unique technique, a very light $\left(\sim 8 \mu \mathrm{g} / \mathrm{cm}^{2}\right)$ graphene coating layer is obtained which does not further imply lowering the entire energy density of the cell. As a whole, the cell with graphene coated paper exhibits $610 \mathrm{mAh} / \mathrm{g}$ discharge capacity at C/5 after 100 cycles, while the cell without interlayer exhibits much poorer performance. The improved performance is mostly associated with the interaction between graphene and polysulfide species which is proven by X-Ray Photoelectron Spectroscopy measurement as well as the excellent electronic conductivity of graphene layer which behaves as a secondary current collector resulting the cell resistance decrease.
\end{abstract}

(C) 2018 Elsevier B.V. All rights reserved.

\section{Introduction}

Lithium-ion batteries (Li-ion) have invaded the marketplace of mobile applications for approximately 30 years which is not only because of the advances in technology, but also relatively high capacity and stability. Yet, the capacity obtained from conventional Liion batteries nearly reached their upmost energy density which offer limited capacities for some specific applications such as electric vehicles. Thus, alternative battery technologies indeed should be investigated, one of which is lithium-sulfur (Li-S) batteries offering high energy density and high theoretical capacity at low cost [1,2].

\footnotetext{
* Corresponding author. Institute of Nanotechnology, Gebze Technical University, 41400, Gebze, Kocaeli, Turkey.

E-mail address: demir-cakan@gtu.edu.tr (R. Demir-Cakan).
}

Since Li-S batteries have several issues that need to be solved, they have not been commercialized until now. These issues can be linked with (i) low electronic conductivity of sulfur, (ii) dissolution of polysulfide species in organic electrolytes, (iii) shuttle effect of dissolved polysulfides and (iv) safety issues due to the use of lithium metal. The conductivity problem can be simply solved by the use of carbon or conductive polymer composites. However, migration of dissolved polysulfide species from cathode to anode side by the effect of electric field or concentration gradient between anode and cathode causes fast capacity fading. In order to overcome $\mathrm{Li}$ anode poisoning from polysulfide species, the use of microporous carbon paper between cathode and separator was proposed [3]. Since then significant progress has been made in the field of interlayers used in Li-S batteries [4,5]. Nafion based [6-9], carbon based [10-13] and polymer based [14-17] interlayers located between anode and cathode electrodes have been 
suggested in the field of Li-S batteries.

Graphene is a very prevalent material with its excellent mechanical and electronic properties which is used in many applications including energy, electronics and biotechnology [18-21]. It offers very high specific surface area and excellent electrical conductivity. In the field of batteries, graphene is used as electrode material [22,23], support material [18,24] or interlayer [25-27]. Up to now, bare graphene [28-32], hybrid graphene composite [33-39] or functionalized graphene [26,40-42] based interlayers have been applied in Li-S batteries. By the help of inserting graphene interlayer, polysulfides can be caught due to the adsorption ability of graphene. The functional groups on the structure also increase the polysulfide adsorption ability. Likewise, the cell resistance can also be reduced with the intimate contact between cathode and graphene interlayer which behave like a secondary current collector.

In literature, various types of graphene based-interlayers were tried in Li-S batteries, as mentioned above. Many of these studies contain blend of graphene/graphene oxide powders and binders to coat on the separator. This perspective unfortunately results in an increase of the weight of the interlayer which causes lowering of the energy density. Within this context, Chemical Vapor Deposition (CVD) enables conformal and thin coating of graphene without binder. Hitherto, CVD grown graphene interlayers in Li-S batteries were subjected in two different studies. Du et al. used commercial CVD grown graphene coating as interlayer [32]. On the other hand, Ou et al. synthesized poly(methyl methacrylate) coated CVD grown graphene on Polypropylene separator [36]. In our work, multilayer graphene was synthesized on nickel foil by CVD and as-prepared graphene was simply transferred to paper separator in pure water that is called fishing technique which enables conformal and facile transfer of coating at a short time.

Herein, the ultra-lightweight multilayer graphene coated paper separator used as an interlayer in Li-S batteries. This coating has resulted almost no additional weight on the paper, thus it does not lower the entire energy density of the cell. In the field of Li-S batteries, the weight of the suggested interlayers is a very critical point and it should not be underestimated, since it imposes a pronounced drawback in terms of energy density to the Li-S system which cannot compete with Li-ion batteries readily available in the marketplace. Indeed, with regards to the other graphene based interlayers studies suggested in the literature (which were summarized in Table S2, Supporting Information), the proposed interlayer has one of the lowest reported weight per selected area due to the unique synthesis and transfer printed technique. As a whole, in comparison to the cell without graphene coating, high electronic conductivity and good polysulfide adsorption ability of the graphene layer indeed resulted in improved electrochemical performance.

\section{Experimental}

\subsection{Preparation of multilayer graphene coatings}

Multilayer graphene samples were synthesized on a $50 \mu \mathrm{m}$ thick nickel foil (Alfa Aesar item \# 12722) by Chemical Vapor Deposition (CVD). To remove the oxide layer on nickel, the foils were annealed at $1000{ }^{\circ} \mathrm{C}$ under $100 \mathrm{sccm} \mathrm{H}_{2}$ and $100 \mathrm{sccm}$ Ar gases. The growth of graphene was carried out at $1000^{\circ} \mathrm{C}$ at the ambient pressure under flow of $\mathrm{H}_{2}$, $\mathrm{Ar}$ and $\mathrm{CH}_{4}$ gases with flow rates of $30 \mathrm{sccm}, 100 \mathrm{sccm}$ and $100 \mathrm{sccm}$, respectively. After 5 minutes growth time, the samples were exposed to room temperature under argon gas flow for the fast cooling.

Multilayer graphene was transfer printed on glass fiber separator (Whatman GF/C) by the "fishing technique". In this technique, the graphene coated nickel foil was immersed into dense iron trichloride solution ( 1 molar), and then it was placed on a surface of the DI water. After that, a piece of paper was submerged into the same container and then pulled out slowly by allowing a conformal coating of multilayer graphene on the surface of the separator (Fig. 1a). Finally, graphene coated papers were dried at $70^{\circ} \mathrm{C}$ for 4 hours under ambient conditions.
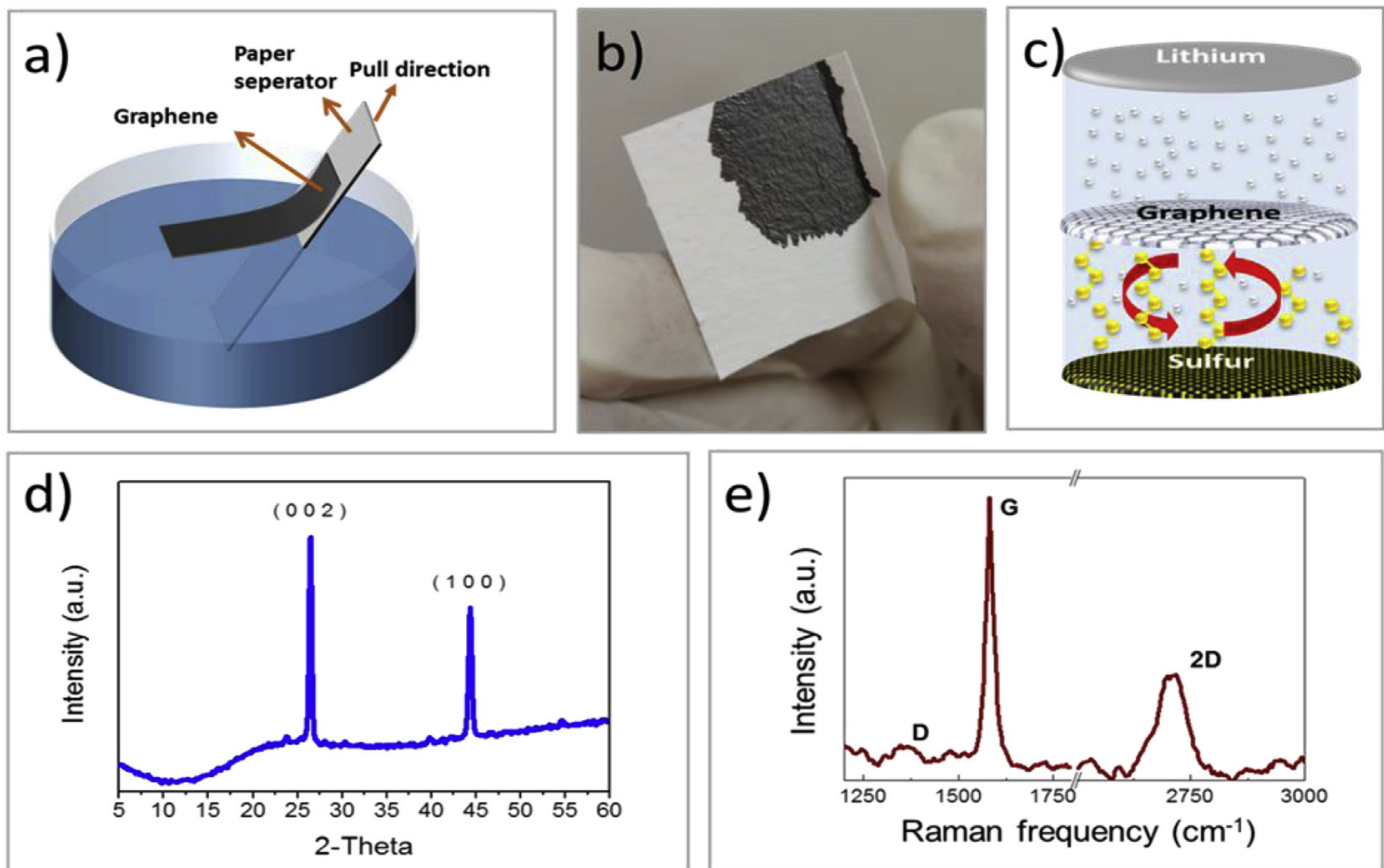

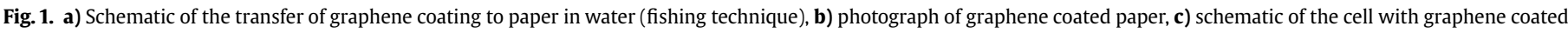
paper, d) X-Ray Diffraction pattern of graphene coating, e) Raman spectrum of graphene coated paper. 


\subsection{Synthesis of $\mathrm{Li}_{2} \mathrm{~S}_{5}$ powder}

Stoichiometric amounts of lithium metal and sulfur was added to ethylene glycol diethyl ether and the as-prepared solution was stirred at $150^{\circ} \mathrm{C}$ for 2 days to obtain $\mathrm{Li}_{2} \mathrm{~S}_{5}$ composition. After drying, $0.1 \mathrm{M} \mathrm{Li}_{2} \mathrm{~S}_{5}$ in 1,3-Dioxolane (DOL) solution was prepared for X-Ray Photoelectron Spectroscopy measurements.

\subsection{Material characterizations}

The X-Ray Diffraction of materials were performed by using Rigaku D-MAX 2200 diffractometry $\left(40 \mathrm{kV}, 40 \mathrm{~mA}\right.$ ), with a $\mathrm{Cu} \mathrm{K}_{\alpha}$ radiation over a $2 \theta$ range from $5^{\circ}$ to $60^{\circ}$ with the scanning rate of $0.5^{\circ} / \mathrm{min}$.

The microstructural-morphology examination and the chemical composition determination of graphene coatings were conducted by SEM FEI/Philips XL30 FEG ESEM equipped with Energy Dispersive X-Ray Spectroscopy (EDS).

Bruker Vertex 70V FTIR spectrometer was used in order to perform the optical transmittance measurements. For this purpose, the multilayer graphene was transfer printed on PVC substrate by using the lamination technique at $120^{\circ} \mathrm{C}$ [43].

To investigate the chemical composition of graphene coating and support the idea of the adsorption of polysulfides by graphene, the X-Ray Photoelectron Spectroscopy (XPS) measurements were conducted using a Phoibos 150 Specs charged particle analyzer. To do so, $0.1 \mathrm{M} \mathrm{Li}_{2} \mathrm{~S}_{5}$ containing 1,3-Dioxolane (DOL) was prepared, then poured over the graphene coated paper and analyzed by XPS. Graphene coated paper without polysulfide treatment was also investigated. A conventional $\mathrm{Al} \mathrm{K}$. radiation $(\mathrm{h} U=1486.61 \mathrm{eV})$ at $300 \mathrm{~W}(15 \mathrm{kV}$ and $20 \mathrm{~mA}$ ) was used. For fitting process of the XPS results, the own adapted macro in Igor 4.0 version was used. The Shirley's function was used to provide the background-subtracted photoemission spectra in order to eliminate the effect of inelastic background noise. Gaussian type functions were used for fitting.

\subsection{Cell assembly and electrochemical measurements}

The cathode electrode was prepared by mixing Sulfur powder and Carbon Ketjen Black. After that, the mixture was heat-treated at $155^{\circ} \mathrm{C}$ for 6 hours in oven (melt diffusion method). The resulting composite contains $63 \mathrm{wt} \%$ sulfur. Thermogravimetric Analysis (TGA) result of the prepared cathode can be seen from Fig. S1. Total sulfur content in the prepared cells was $\sim 3 \mathrm{mg} / \mathrm{cm}^{2}$.

Two electrode Swagelok ${ }^{\mathrm{TM}}$ cells were assembled in a high purity argon containing glove box (Innovative Technology, USA). Lithium metal foil was used as both counter and reference electrode. $1 \mathrm{M}$ LiTFSI (Lithium bis(trifluoromethanesulfonyl)imide, from SigmaAldrich) with $0.5 \mathrm{M} \mathrm{LiNO}_{3}$ (Lithium nitrate, from Acros) containing 1,3-Dioxolane: 1,2-Dimethoxyethane (DOL:DME) solvent was used as electrolyte. Electrolyte/sulfur ratio was kept at $30 \mu \mathrm{L} / \mathrm{mg}$. Graphene coated separator was placed between anode and cathode electrode. The bare paper separator weighed as $4.7 \mathrm{mg} / \mathrm{cm}^{2}$ and the thickness and the weight of the synthesized graphene were estimated as 100 layers [43] and $\sim 8 \mathrm{~g} / \mathrm{cm}^{2}$, respectively, meaning that additional weight from graphene coating is negligible for the calculation of energy density. Even though the areal weightiness of the Li-ion commercial separator $\left(1.4 \mathrm{mg} / \mathrm{cm}^{2}\right)$ is much lower than that of paper separator from Whatmann $\left(4.7 \mathrm{mg} / \mathrm{cm}^{2}\right)$, we have concluded in our former study that better cycling capabilities could be achieved with paper separators in Li-S cells [2]. The schematic of the cell can be seen from Fig. 1c in which graphene coated separator was placed between anode and cathode electrodes.

The discharge/charge tests were performed at $\mathrm{C} / 5$ (1C corresponds to a current density to reach the theoretical capacity in 1 hour) between $1.9 \mathrm{~V}-3 \mathrm{~V}\left(\mathrm{Vs} \mathrm{Li}^{+} / \mathrm{Li}\right)$ by using VMP3-Biologic galvanostat/potentiostat at room temperature.

Cycling voltammetry (CV) was conducted with a scan rate of $0.1 \mathrm{mV} / \mathrm{s}$ between $1.7 \mathrm{~V}-3 \mathrm{~V}$ by using VMP3-Biologic galvanostat/ potentiostat.

Electrochemical Impedance Spectra (EIS) of lithium electrode was carried out using a three electrode cell in which lithium was used as a reference electrode placed on a 316L grade stainless steel wire. Both working and reference electrodes were lithium. The measurement was performed within the range of $200 \mathrm{kHz}$ to $2 \mathrm{mHz}$, with a voltage amplitude of $5 \mathrm{mV}$ around $0 \mathrm{~V}\left(\mathrm{Li}^{+} / \mathrm{Li}\right)$ in VMP3. The spectra of the lithium electrode was collected at the open circuit voltage (OCV) for every 2 hours of interval time. In order to monitor and compare the change of resistance over time, two different cell combinations were prepared; the one without interlayer and the other one is the cell with graphene coated paper.

\section{Results and Discussion}

The schematic procedure of the conformal coating of graphene on the surface of the paper separator and the photograph of the prepared interlayer can be seen at Fig. 1a and b, respectively. During the electrochemical characterizations, graphene coated separator was placed between anode and cathode electrodes as illustrated in Fig. 1c. Fig. 1d shows the X-Ray Diffraction pattern of the separator after graphene coating. The sharp peak located at $2 \theta=26^{\circ}$ corresponds to the diffraction of (002) plane with a d-spacing of $3.34 \AA$ and the peak at $2 \Theta=44^{\circ}$ can be attributed to the (100) plane of graphene. These peaks show characteristic highly ordered structure of graphene layers. Raman spectrum of the multilayer graphene (Fig. 1e) shows typical spectra (the intensity ratio of $2 \mathrm{D} / \mathrm{G}$ is 3.3 and the intensity of the defect mode (D) is negligible.) which ensures that the quality of the graphene is very high.

Scanning Electron Microscopy was conducted to characterize the morphology of graphene coated paper separator. Fig. 2a shows the bare paper separator before graphene coating. After graphene coating, the fibrous structure is totally disappeared and the smooth graphene layer can be observed (Fig. 2b). The cross-sectional SEM has also been tempted to be contacted which can be seen at the inset of Fig. 2b. Though a smooth continuous coating layer can be observed at the top of the paper separator, the layer thickness cannot be detected due to the low resolution of the SEM image. Carbon signals which were obtained by Energy Dispersive X-Ray Spectroscopy (EDS) from graphene coating are shown in Fig. 2c.

In order to know the thickness of the coating layer, the optical transmittance measurements were performed (Fig. 2d). To do so, the multilayer graphene was transfer printed on PVC substrate by using the lamination technique at $120^{\circ} \mathrm{C}$ [43]. The optical transmission spectra of the multilayer graphene (Fig. 2d inset) was calculated according to the formula $\mathrm{T}=(1-\alpha)^{\mathrm{N}}$, where $\mathrm{T}$ is the transmission, $\mathrm{N}$ is number of graphene layer and $\alpha$ is the absorption of single layer graphene which is around 2.3\%. Optical measurements showed that the multilayer graphene has $12 \%$ transmittance. Therefore (by using Fig. $2 \mathrm{~d}$ inset), one can find that the number of graphene is around 100 layers. Single layer graphene is $0.34 \mathrm{~nm}$. The thickness of 100 layers of graphene is a little bit more than $34 \mathrm{~nm}$, because the growth and transfer procedures result in some residues on the graphene samples. Since the mass density of graphene is theoretically calculated to be $\sim 76 \mathrm{ng} / \mathrm{cm}^{2}$, the weight of the 100 layers would be around $\sim 8 \mathrm{~g} / \mathrm{cm}^{2}$. Moreover, the sheet resistance of the 100 layers graphene was found to be around $240 \Omega / s q$ [19].

To understand the chemical structure of graphene coating and to support the idea of polysulfide adsorption effect of graphene, XRay Photoelectron Spectroscopy (XPS) analysis was applied. To 

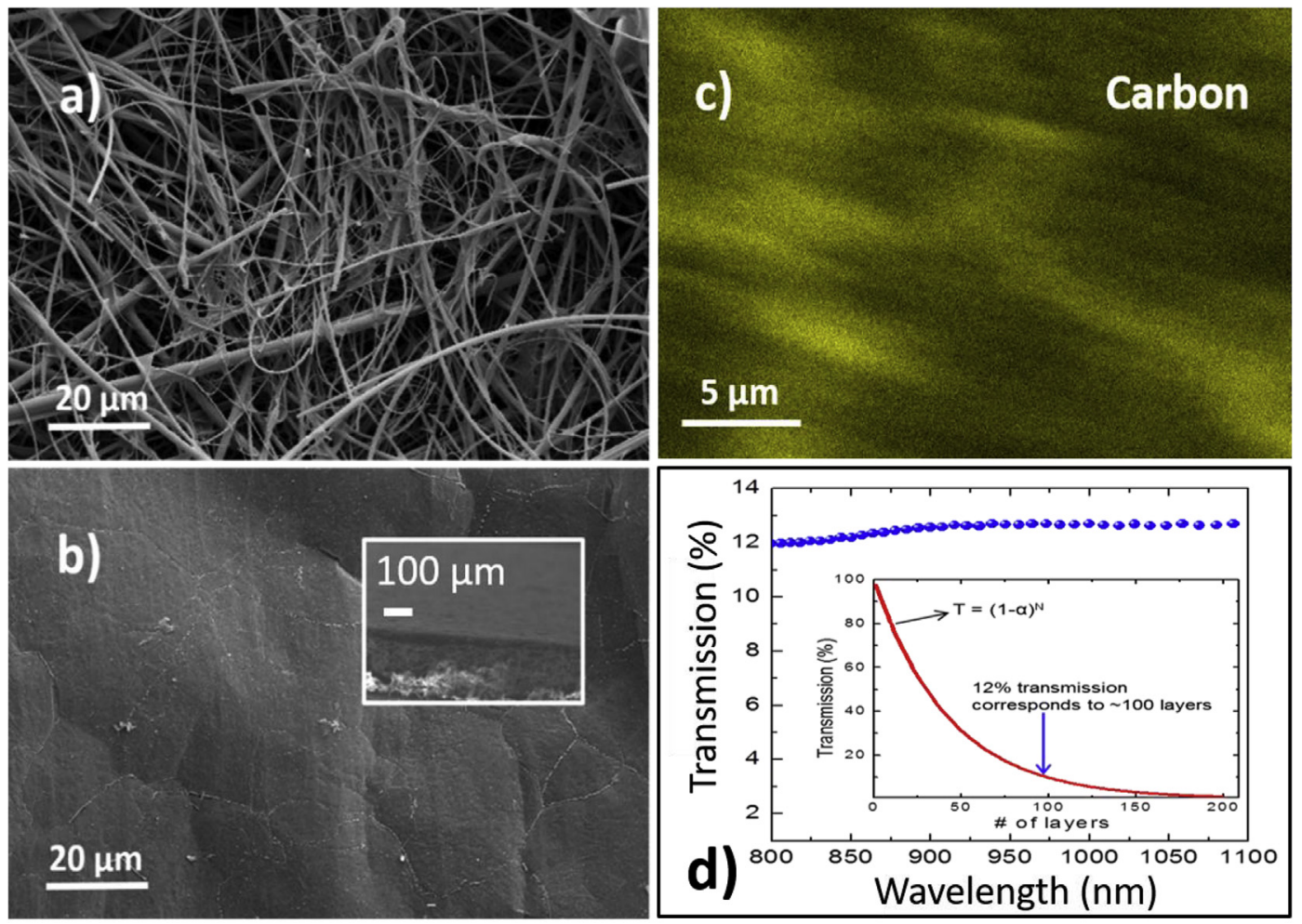

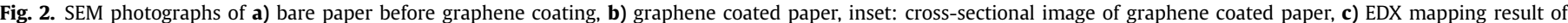

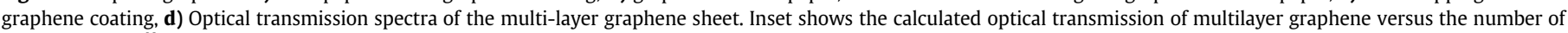
layers. $T=(1-\alpha)^{\mathrm{N}}$, where $\mathrm{T}$ is the transmission, $\mathrm{N}$ is number of graphene layers and $\alpha$ is the absorption of the single layer graphene which is around $2.3 \%$.

underline the adsorption phenomena, $0.1 \mathrm{M} \mathrm{Li}_{2} \mathrm{~S}_{5}$ containing DOL solution was prepared and dropped on a graphene coated paper. After drying procedure, this sample was investigated by XPS. Untreated graphene coated paper has also been examined for comparison and for understanding the chemical structure. Fig. 3a shows the $\mathrm{C} 1 \mathrm{~s}$ spectrum of the graphene coated paper. $\mathrm{C} 1 \mathrm{~s}$ spectrum deconvoluted into four peaks at around $284.85 \mathrm{eV}, 286 \mathrm{eV}$, $287.1 \mathrm{eV}$ and $289.8 \mathrm{eV}$ which resemble C-C in $\mathrm{sp}^{2}$-hybridized domains, hydroxyl groups $(\mathrm{C}-\mathrm{OH})$, carbonyl $(\mathrm{C}=\mathrm{O})$ and carboxyl groups $(\mathrm{O}=\mathrm{C}-\mathrm{O})$. These functional groups are known for their ability to capture polysulfides and help them to stay at the cathode side by forming Li-O bonds throughout their oxygenated groups and lithium in polysulfides. Indeed, when $\mathrm{Li}_{2} \mathrm{~S}_{5}$-containing DOL was poured on graphene coating, it can be clearly seen that there is a huge shift in binding energy which is an indicator of adsorption. XPS result of $\mathrm{Li}_{2} \mathrm{~S}_{5}$-containing DOL solution poured graphene coated paper can be seen from Fig. 3b. C1s spectrum of polysulfide treated graphene coating also deconvoluted into four peaks like bare graphene coated paper. Binding energy of C-C shifted from $284.85 \mathrm{eV}$ to $286.25 \mathrm{eV}, \mathrm{C}-\mathrm{OH}$ groups shifted from $286 \mathrm{eV}$ to $287.1 \mathrm{eV}, \mathrm{C}=\mathrm{O}$ groups shifted from $287.1 \mathrm{eV}$ to $289.15 \mathrm{eV}$ and $\mathrm{O}=\mathrm{C}-$ $\mathrm{O}$ groups shifted from $289.8 \mathrm{eV}$ to $292.3 \mathrm{eV}$. These huge shifts show the strong adsorption ability of graphene coating by its functional groups.

Fig. 4 compares the cycling voltammetry $(\mathrm{CV})$ results of the Li-S cells with only bare paper separator and graphene coated paper, cycled between $1.7 \mathrm{~V}$ and $3.0 \mathrm{~V}$ at a scan rate of $0.1 \mathrm{mV} / \mathrm{s}$. Fig. $4 \mathrm{a}$ represents the cell without interlayer and Fig. 4b shows the cell with graphene coated paper. Both of the cells show two reduction peaks in the cathodic scans at approximately $2.25 \mathrm{~V}$ and $1.95 \mathrm{~V}$ which represent the typical conversion from elemental sulfur to long-chain polysulfides and long chain polysulfides to low-chain

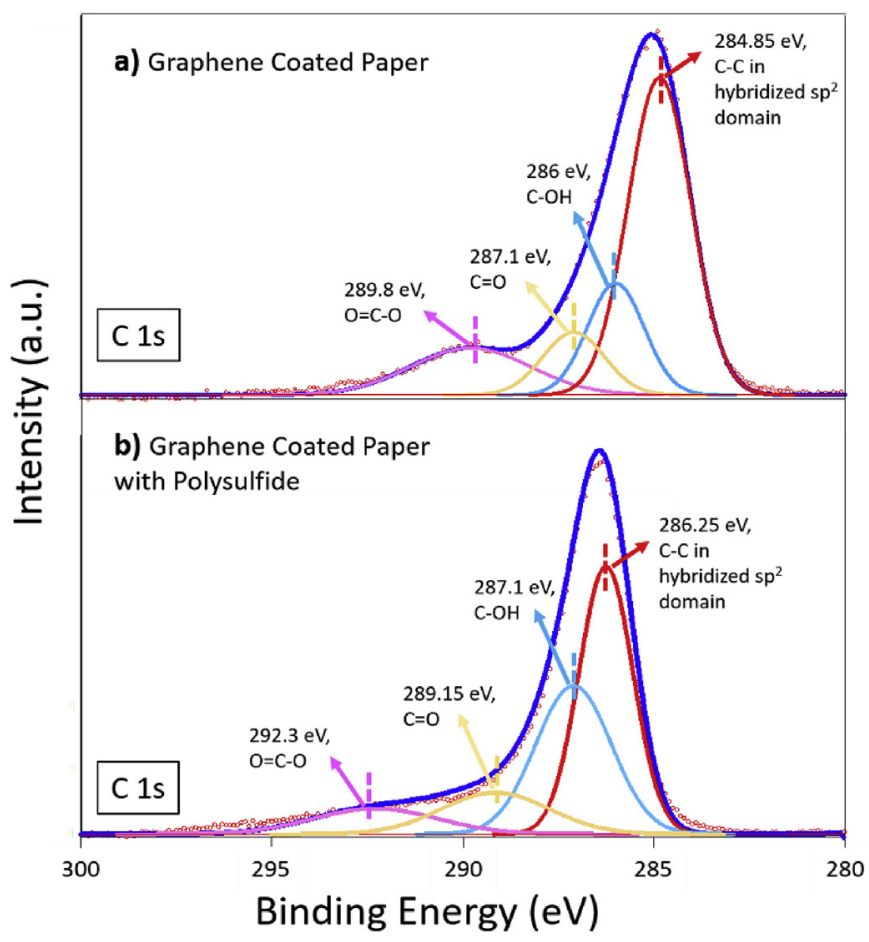

Fig. 3. X-Ray Photoelectron Spectroscopy results, high resolution scan of $C 1 \mathrm{~s}$. a) Graphene coated paper, b) polysulfide dropped graphene coated paper. 

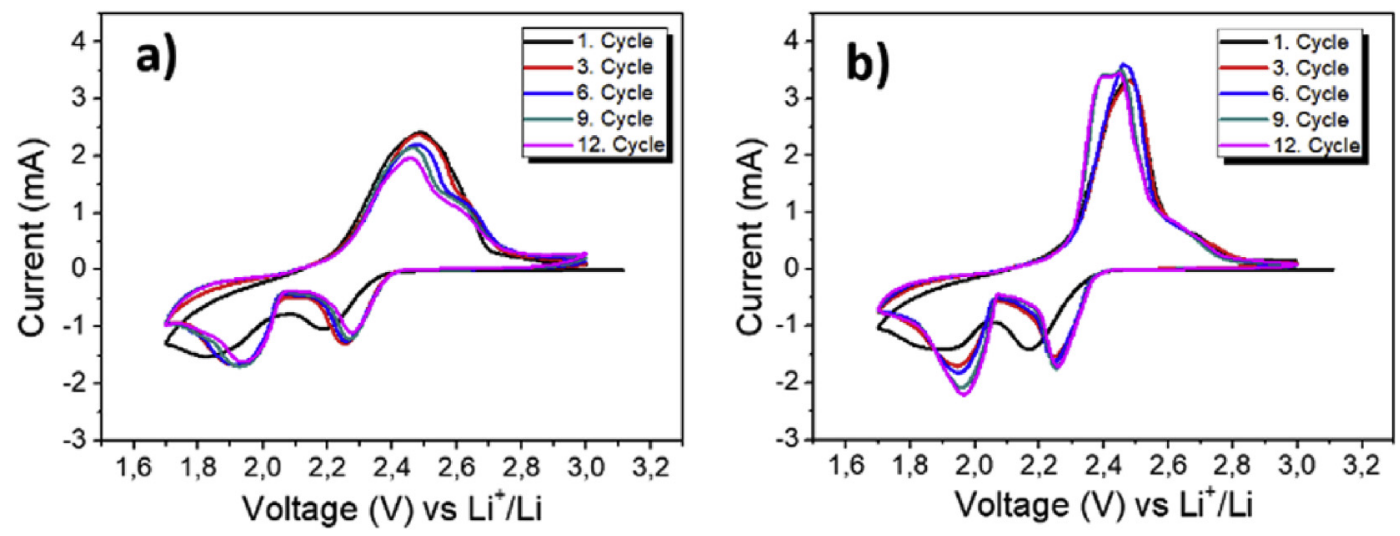

Fig. 4. Cycling Voltammetry (CV) results of a) without interlayer and b) with graphene coated paper at a scan rate of $0.1 \mathrm{mV} / \mathrm{s}$.
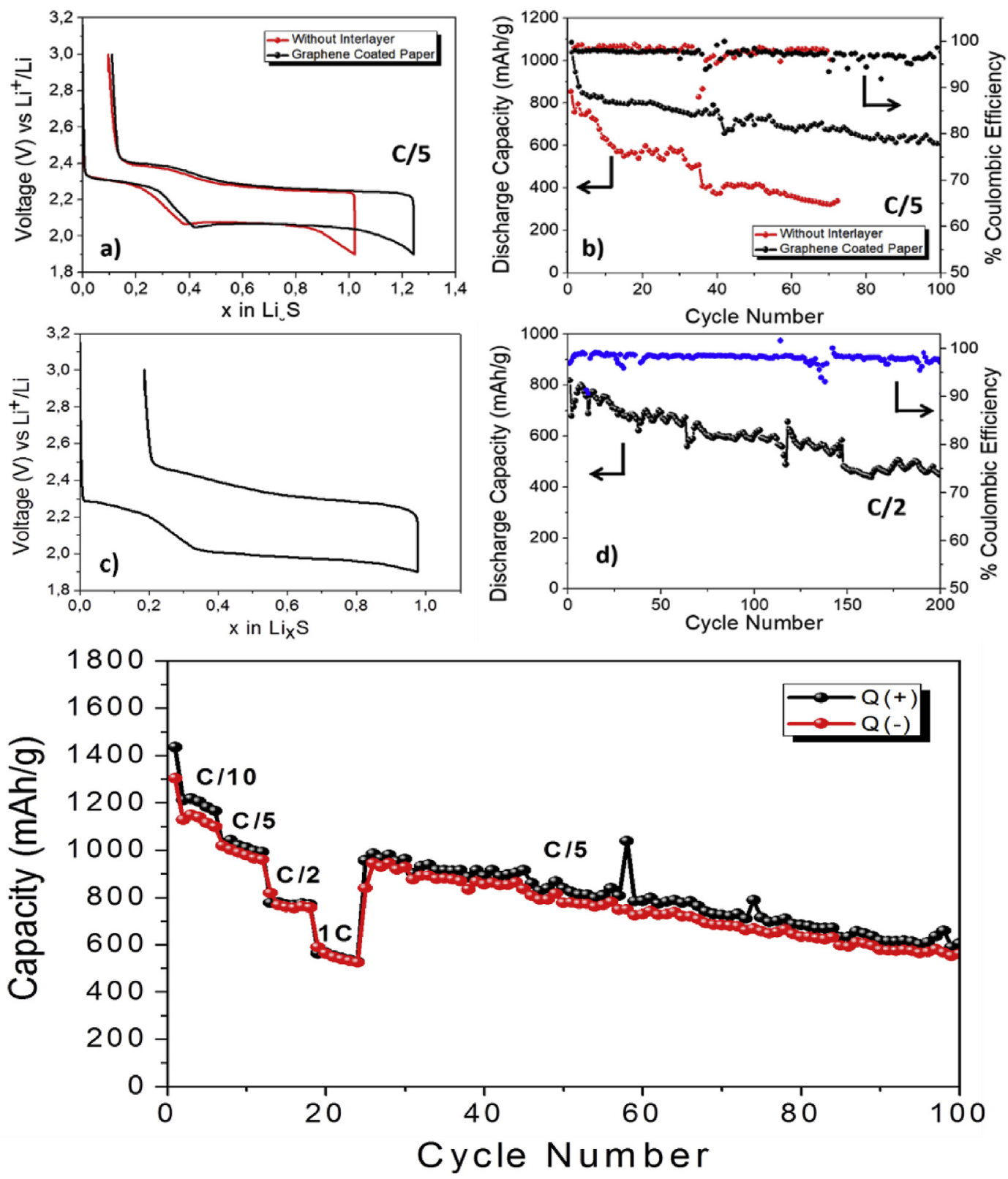

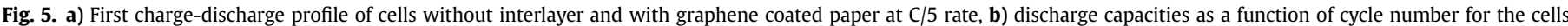

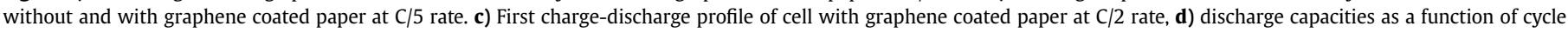
number for the cell with graphene coated paper at $\mathrm{C} / 2$ rate. e) Rate capability of the cell with graphene coated paper. 


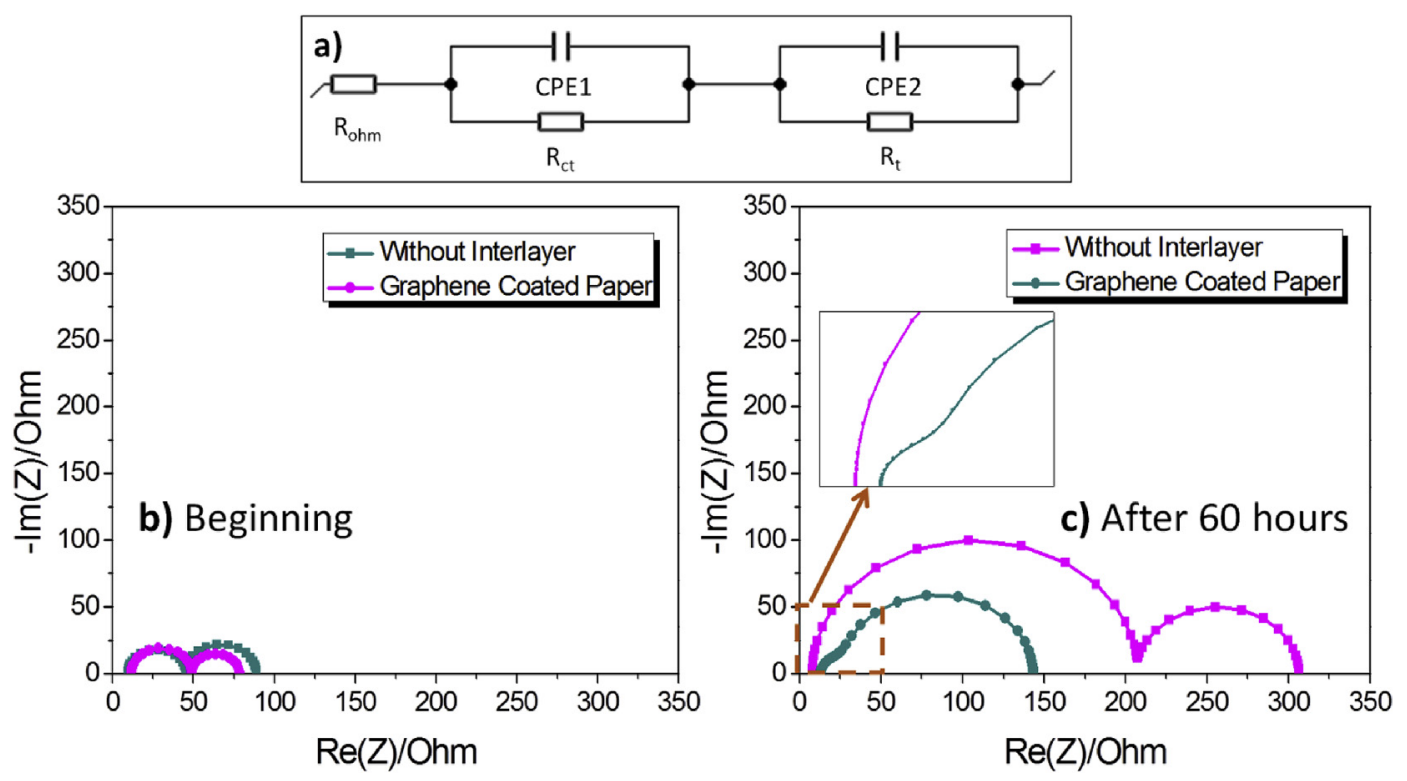

Fig. 6. EIS spectra of the cells without interlayer and with graphene coated paper. a) the fitting equivalent circuit, b) at the beginning, c) after 60 hours.

polysulfides, respectively. The intensity of the peaks without interlayer decreased as the cycle number increased. On the other hand, after insertion of the graphene coated paper, a sustained cycling is observed which may be associated with the increased ion and electron transport enabled by graphene coating [27]. In addition to this, the cell with graphene coating has higher current peaks with respect to the cell without interlayer which is a sign of high electrochemical kinetics and better utilization of the active materials [35]. These results show the effectivity of inserting graphene coating resulting of high electrochemical stability and reversibility.

To further show the effectivity of addition of graphene coated paper, electrochemical cycling tests were conducted. To do so, the cell with and without graphene coated paper were prepared and tested simultaneously at a current rate of $\mathrm{C} / 5$ (1C corresponds to a current density to reach the theoretical capacity in 1 hour) between $1.9 \mathrm{~V}$ and $3.0 \mathrm{~V}$. The reason for working in narrow cut-off voltage is to avoid decomposition of $\mathrm{LiNO}_{3}$ under $1.9 \mathrm{~V}$ [44]. The results are shown in Fig. 5a and Fig. 5b which represent charge-discharge profile of the cells and discharge capacity as a function of cycle number, respectively. Both of the cells show typical two discharge plateaus. The higher plateau located at $2.3 \mathrm{~V}$ indicates the transition of sulfur to high-order polysulfides and the lower plateau located at $2.0 \mathrm{~V}$ indicates the transition of high-order polysulfides to loworder polysulfides (Fig. 5a). The cell with graphene coating exhibits $1039 \mathrm{mAh} / \mathrm{g}$ discharge capacity at initial cycle and after 50 cycles $703 \mathrm{mAh} / \mathrm{g}$ discharge capacity is obtained. On the other hand, the cell without interlayer has $854 \mathrm{mAh} / \mathrm{g}$ discharge capacity at first cycle and $396 \mathrm{mAh} / \mathrm{g}$ discharge capacity was obtained after 50 cycles which was terminated after 76 cycles due to the shuttle effect. Additionally, fluctuation of the coulombic efficiency in Fig. $5 \mathrm{~b}$ is slightly lessen in the presence of graphene interlayer. The long term cycling stability of the cell with graphene coated paper at higher current rate is shown in Fig. $5 c$ and $d$. The cell cycled at $\mathrm{C} / 2$ exhibited $817 \mathrm{mAh} / \mathrm{g}$ initial discharge capacity and $450 \mathrm{mAh} / \mathrm{g}$ discharge capacity was obtained after 200 cycles which show that the cell preserved $55 \%$ of its capacity.

The rate capability test of the cell with graphene coated paper is presented in Fig. 5e. The cell was started to cycle at low rates which then was increased up to $1 \mathrm{C}$. The cell exhibited $1303 \mathrm{mAh} / \mathrm{g}$ discharge capacity at the first cycle, then it decreased to $1115 \mathrm{mAh} / \mathrm{g}$ at C/10. The discharge capacity decreased to $960 \mathrm{mAh} / \mathrm{g}, 760 \mathrm{mAh} / \mathrm{g}$ and $524 \mathrm{mAh} / \mathrm{g}$ as the current rate was increased to $\mathrm{C} / 5, \mathrm{C} / 2$ and $1 \mathrm{C}$, respectively. When the current rate was returned back to $C / 5$, the cell recovered its capacity and exhibited $\sim 950 \mathrm{mAh} / \mathrm{g}$ discharge capacity. When all the above mentioned electrochemical results are taken into account, indeed it demonstrates that graphene coating enables better cycling stability and electrochemical performance. These improved results can be associated with the excellent electronic conductivity of graphene coated paper and its good adsorption ability to catch polysulfides.

Graphene is known to have excellent electronic conductivity which is expected to end with lowering the cell resistance. Electrochemical impedance spectroscopy (EIS) was performed to monitor the solid electrolyte interface (SEI) growth on the Li electrode surface. The cell without interlayer was also investigated for the sake of comparison. To do so, three electrode cells were used and impedance spectra was recorded for every two hours of interval at open-circuit conditions without applying voltage. Fig. 6 shows the Nyquist plots of the cells at the beginning with the fresh samples (Fig. 6b) and after 60 hours aging (Fig. 6c). The fitting equivalent circuit is shown in Fig. 6a. Both of the cells show two semicircles at high to medium frequency showing charge transfer resistance $\left(R_{c t}\right)$ and medium to low frequency presenting resistance from passivating film $\left(R_{t}\right)$. $R_{\text {ohm }}$ presents the ohmic losses. The results obtained from fitted Electrochemical Impedance Spectra can be seen from Table S2. In both cases, the resistance of the surface layer increased upon resting time due to the interaction between highly reactive Li metal and the electrolyte solvent, salts and in-situ formed polysulfides. However, the resistance difference of the cell with graphene coating is much lower upon 60 hours of rest time than that of the cell without interlayer. These results meet the expectations and it can be attributed to the improved electrode/ electrolyte interface and increased charge transfer due to the addition of highly conductive graphene coated paper.

\section{Conclusions}

Even though the use of interlayer concept is well recognized by the Li-S battery system, lowering the entire energy density due to the thick and heavy-weight coating layer is one of the 
shortcomings. Herein, ultra-lightweight $\left(\sim 8 \mu \mathrm{g} / \mathrm{cm}^{2}\right)$ CVD grown multilayered graphene coated paper was used both for the adsorbing of polysulfides and for improving the electrochemical performance. Graphene was firstly coated on nickel foil and thereafter it was transfer printed to the paper separator by fishing technique. The interlayer was placed between anode and cathode electrodes and better performance was obtained with respect to the cell without interlayer. The good performance is associated with the better electronic wiring and polysulfide adsorption ability of graphene. Electrochemical Impedance Spectroscopy (EIS) results demonstrates reduced cell resistance by the use of graphene coated paper due to its excellent electronic conductivity. The adsorption ability of graphene layer was supported by X-Ray Photoelectron Spectroscopy (XPS) measurement which shows an obvious shift in binding energies in the presence of polysulfides. The functional groups in graphene structure may also help in increasing electrochemical performance as supported by XPS analysis. As a whole, $610 \mathrm{mAh} / \mathrm{g}$ discharge capacity was obtained after 100 cycles at C/5 rate which is much better than the cell without interlayer.

\section{Conflicts of interest}

The authors declare that there is no conflict of interests.

\section{Acknowledgements}

RDC gratefully thanks The Scientific and Technological Research Council of Turkey (TÜBITAK) for partial financial support (Project No. 213M374/National Young Researchers Career Development Grant). The authors would like to express their thanks to Ahmet NAZIM for SEM and Adem SEN for XRD measurements. This work is a part of doctorate thesis of Elif Ceylan Cengiz.

\section{Appendix A. Supplementary data}

Supplementary data to this article can be found online at https://doi.org/10.1016/j.jallcom.2018.11.071.

\section{References}

[1] R. Demir-Cakan, Li-s Batteries: the Challenges, Chemistry, Materials, and Future Perspectives, World Scientific Publishing Company, 2017.

[2] O. Salihoglu, R. Demir-Cakan, Factors affecting the proper functioning of a $3 \mathrm{Ah}$ Li-S pouch cell, J. Electrochem. Soc. 164 (2017) A2948-A2955.

[3] Y.S. Su, A. Manthiram, Lithium-sulphur batteries with a microporous carbon paper as a bifunctional interlayer, Nat. Commun. 3 (2012).

[4] W. Tianyi, K. Katja, C. Sinho, P. Huan, X. Huaiguo, W. Guoxiu, Fabrication methods of porous carbon materials and separator membranes for lithium-sulfur batteries: development and future perspectives, Small Methods 1 (2017), 1700089.

[5] J.-Q. Huang, Q. Zhang, F. Wei, Multi-functional separator/interlayer system for high-stable lithium-sulfur batteries: progress and prospects, Energy Storage Mater. 1 (2015) 127-145.

[6] I. Bauer, S. Thieme, J. Brückner, H. Althues, S. Kaskel, Reduced polysulfide shuttle in lithium-sulfur batteries using Nafion-based separators, J. Power Sources 251 (2014) 417-422.

[7] Z. Jin, K. Xie, X. Hong, Z. Hu, X. Liu, Application of lithiated Nafion ionomer film as functional separator for lithium sulfur cells, J. Power Sources 218 (2012) $163-167$.

[8] X. Yu, J. Joseph, A. Manthiram, Polymer lithium-sulfur batteries with a Nafion membrane and an advanced sulfur electrode, J. Mater. Chem. A 3 (2015) $15683-15691$.

[9] T.-Z. Zhuang, J.-Q. Huang, H.-J. Peng, L.-Y. He, X.-B. Cheng, C.-M. Chen, Q. Zhang, Rational integration of polypropylene/graphene oxide/nafion as ternary-layered separator to retard the shuttle of polysulfides for lithium-sulfur batteries, Small 12 (2016) 381-389.

[10] J. Balach, T. Jaumann, M. Klose, S. Oswald, J. Eckert, L. Giebeler, Mesoporous carbon interlayers with tailored pore volume as polysulfide reservoir for highenergy lithium-sulfur batteries, J. Phys. Chem. C 119 (2015) 4580-4587.

[11] L.-B. Xing, K. Xi, Q. Li, Z. Su, C. Lai, X. Zhao, R.V. Kumar, Nitrogen, sulfurcodoped graphene sponge as electroactive carbon interlayer for high-energy and -power lithium-sulfur batteries, J. Power Sources 303 (2016) 22-28.
[12] J. Zhang, H. Li, Z. Lin, Q. Tang, W. Qi, L. Wang, H. Zheng, K. Zhou, A conductive carbon interlayer modified by magnetron sputtering for improvedperformance lithium-sulfur batteries, RSC Adv. 7 (2017) 39172-39177.

[13] K.B. Celik, E.C. Cengiz, T. Sar, B. Dursun, O. Ozturk, M.Y. Akbas, R. Demir-Cakan, In-situ wrapping of tin oxide nanoparticles by bacterial cellulose derived carbon nanofibers and its application as freestanding interlayer in lithium sulfide based lithium-sulfur batteries, J. Colloid Interface Sci. 530 (2018) $137-145$.

[14] J.-Q. Huang, Q. Zhang, S.-M. Zhang, X.-F. Liu, W. Zhu, W.-Z. Qian, F. Wei, Aligned sulfur-coated carbon nanotubes with a polyethylene glycol barrier at one end for use as a high efficiency sulfur cathode, Carbon 58 (2013) 99-106.

[15] G. Ma, Z. Wen, Q. Wang, C. Shen, P. Peng, J. Jin, X. Wu, Enhanced performance of lithium sulfur battery with self-assembly polypyrrole nanotube film as the functional interlayer, J. Power Sources 273 (2015) 511-516.

[16] N. Yan, X. Yang, W. Zhou, H. Zhang, X. Li, H. Zhang, Fabrication of a nano-Li+channel interlayer for high performance Li-S battery application, RSC Adv. 5 (2015) 26273-26280.

[17] S.S. Zhang, D.T. Tran, Z. Zhang, Poly(acrylic acid) gel as a polysulphide blocking layer for high-performance lithium/sulphur battery, J. Mater. Chem. A 2 (2014) 18288-18292.

[18] B. Dursun, E. Topac, R. Alibeyli, A. Ata, O. Ozturk, R. Demir-Cakan, Fast microwave synthesis of SnO2@graphene/N-doped carbons as anode materials in sodium ion batteries, J. Alloys Compd. 728 (2017) 1305-1314.

[19] E.O. Polat, H.B. Uzlu, O. Balci, N. Kakenov, E. Kovalska, C. Kocabas, Grapheneenabled optoelectronics on paper, ACS Photonics 3 (2016) 964-971.

[20] O. Salihoglu, S. Balci, C. Kocabas, Plasmon-polaritons on graphene-metal surface and their use in biosensors, Appl. Phys. Lett. 100 (2012), 213110.

[21] M.S. Yazici, M.A. Azder, O. Salihoglu, CVD grown graphene as catalyst for acid electrolytes, Int. J. Hydrogen Energy 43 (23) (2018) 10710-10716. https://doi. org/10.1016/j.ijhydene.2018.01.180.

[22] J. Hassoun, F. Bonaccorso, M. Agostini, M. Angelucci, M.G. Betti, R. Cingolani, M. Gemmi, C. Mariani, S. Panero, V. Pellegrini, B. Scrosati, An advanced lithium-ion battery based on a graphene anode and a lithium iron phosphate cathode, Nano Lett. 14 (2014) 4901-4906.

[23] G. Wang, X. Shen, J. Yao, J. Park, Graphene nanosheets for enhanced lithium storage in lithium ion batteries, Carbon 47 (2009) 2049-2053.

[24] P. Lian, J. Wang, D. Cai, L. Ding, Q. Jia, H. Wang, Porous SnO2@C/graphene nanocomposite with 3D carbon conductive network as a superior anode material for lithium-ion batteries, Electrochim. Acta 116 (2014) 103-110.

[25] Z. Xiao, Z. Yang, L. Wang, H. Nie, M. Zhong, Q. Lai, X. Xu, L. Zhang, S. Huang,

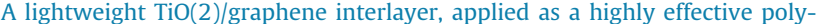
sulfide absorbent for fast, long-life lithium-sulfur batteries, Adv. Mater. 27 (2015) 2891-2898.

[26] H. Deng, L. Yao, Q.-A. Huang, Q. Su, J. Zhang, G. Du, Highly improved electrochemical performance of Li-S batteries with heavily nitrogen-doped threedimensional porous graphene interlayers, Mater. Res. Bull. 84 (2016) 218-224.

[27] V.C. Hoang, V. Do, I.W. Nah, C. Lee, W.I. Cho, I.H. Oh, Facile coating of graphene interlayer onto Li2S as a high electrochemical performance cathode for lithium sulfur battery, Electrochim. Acta 210 (2016) 1-6.

[28] A. Vizintin, M.U.M. Patel, B. Genorio, R. Dominko, Effective separation of lithium anode and sulfur cathode in lithium-sulfur batteries, ChemElectroChem 1 (2014) 1040-1045.

[29] X. Wang, Z. Wang, L. Chen, Reduced graphene oxide film as a shuttleinhibiting interlayer in a lithium-sulfur battery, J. Power Sources 242 (2013) 65-69.

[30] Y. Guo, G. Zhao, N. Wu, Y. Zhang, M. Xiang, B. Wang, H. Liu, H. Wu, Efficient synthesis of graphene nanoscrolls for fabricating sulfur-loaded cathode and flexible hybrid interlayer toward high-performance Li-S batteries, ACS Appl. Mater. Interfaces 8 (2016) 34185-34193.

[31] P.-Y. Zhai, H.-J. Peng, X.-B. Cheng, L. Zhu, J.-Q. Huang, W. Zhu, Q. Zhang, Scaled-up fabrication of porous-graphene-modified separators for highcapacity lithium-sulfur batteries, Energy Storage Mater. 7 (2017) 56-63.

[32] Z. Du, C. Guo, L. Wang, A. Hu, S. Jin, T. Zhang, H. Jin, Z. Qi, S. Xin, X. Kong, Y.G. Guo, H. Ji, L.-J. Wan, Atom-thick interlayer made of CVD-grown graphene film on separator for advanced lithium-sulfur batteries, ACS Appl. Mater. Interfaces 9 (2017) 43696-43703.

[33] G. Zhou, L. Li, D.W. Wang, X.y. Shan, S. Pei, F. Li, H.M. Cheng, A flexible sulfurgraphene-polypropylene separator integrated electrode for advanced $\mathrm{Li}-\mathrm{S}$ batteries, Adv. Mater. 27 (2015) 641-647.

[34] Q. Zeng, X. Leng, K.-H. Wu, I.R. Gentle, D.-W. Wang, Electroactive cellulosesupported graphene oxide interlayers for Li-S batteries, Carbon 93 (2015) $611-619$.

[35] L. Chai, J. Wang, H. Wang, L. Zhang, W. Yu, L. Mai, Porous carbonized graphene-embedded fungus film as an interlayer for superior Li-S batteries, Nano Energy 17 (2015) 224-232.

[36] X. Ou, Y. Yu, R. Wu, A. Tyagi, M. Zhuang, Y. Ding, I.H. Abidi, H. Wu, F. Wang, Z. Luo, Shuttle suppression by polymer-sealed graphene-coated Polypropylene separator, ACS Appl. Mater. Interfaces 10 (2018) 5534-5542.

[37] W. Sun, X. Ou, X. Yue, Y. Yang, Z. Wang, D. Rooney, K. Sun, A simply effective double-coating cathode with $\mathrm{MnO} 2$ nanosheets/graphene as functionalized interlayer for high performance lithium-sulfur batteries, Electrochim. Acta 207 (2016) 198-206.

[38] H. Wu, Y. Huang, W. Zhang, X. Sun, Y. Yang, L. Wang, M. Zong, Lock of sulfur with carbon black and a three-dimensional graphene@carbon nanotubes 
coated separator for lithium-sulfur batteries, J. Alloys Compd. 708 (2017) $743-750$.

[39] H. Li, L. Sun, Y. Zhang, T. Tan, G. Wang, Z. Bakenov, Enhanced cycle performance of Li/S battery with the reduced graphene oxide/activated carbon functional interlayer, J. Energy Chem. 26 (2017) 1276-1281.

[40] C.-Y. Fan, H.-H. Li, L.-L. Zhang, H.-Z. Sun, X.-L. Wu, H.-M. Xie, J.-P. Zhang, Fabrication of functionalized polysulfide reservoirs from large graphene sheets to improve the electrochemical performance of lithium-sulfur batteries, Phys. Chem. Chem. Phys. 17 (2015) 23481-23488.

[41] L. Wang, Z. Yang, H. Nie, C. Gu, W. Hua, X. Xu, X.a. Chen, Y. Chen, S. Huang, A lightweight multifunctional interlayer of sulfur-nitrogen dual-doped graphene for ultrafast, long-life lithium-sulfur batteries, J. Mater. Chem. A 4 (2016) 15343-15352.

[42] H. Wu, Y. Huang, S. Xu, W. Zhang, K. Wang, M. Zong, Fabricating threedimensional hierarchical porous N-doped graphene by a tunable assembly method for interlayer assisted lithium-sulfur batteries, Chem. Eng. J. 327 (2017) 855-867.

[43] E.O. Polat, O. Balcı, C. Kocabas, Graphene based flexible electrochromic devices, Sci. Rep. UK 4 (2014) 6484.

[44] A. Rosenman, R. Elazari, G. Salitra, E. Markevich, D. Aurbach, A. Garsuch, The effect of interactions and reduction products of LiNO3, the anti-shuttle agent in Li-S battery systems, J. Electrochem. Soc. 162 (2015) A470-A473. 